\title{
STUDY OF DIASTOLIC DYSFUNCTION IN YOUNG (< 40 YEARS) NEWLY DETECTED DIABETES AND ITS CORRELATION WITH OBESITY, GLYCAEMIC LEVELS AND DYSLIPIDAEMIA
}

\author{
Ram Anil Raj M. Rㄴ, Yogesh K. Kothari², Kumar Kenchappa ${ }^{3}$, Narayan Raju4, Rajiv Girdhar ${ }^{5}$
}

${ }_{1}^{1}$ Assistant Professor, Department of Cardiology, Rajarajeswari Medical College and Hospital, Bengaluru. 2 Professor and HOD, Department of Cardiology, Rajarajeswari Medical College and hospital, Bengaluru. ${ }^{3}$ Assistant Professor, Department of Cardiology, Rajarajeswari Medical College and hospital, Bengaluru. ${ }_{4}^{4}$ Assistant Professor, Department of Cardiology, Rajarajeswari Medical College and hospital, Bengaluru. ${ }_{5}^{5}$ Senior Resident, Department of Cardiology, Rajarajeswari Medical College and hospital, Bengaluru.

\section{ABSTRACT}

\section{BACKGROUND}

Left Ventricular Diastolic Dysfunction (LVDD) represents the first stage of diabetic cardiomyopathy preceding changes in systolic function, reinforcing the importance of early examination of ventricular function in individuals with diabetes mellitus (DM). This cross-sectional study was conducted to determine the incidence of asymptomatic LVDD in young (less than 40 years) and newly diagnosed normotensive cases of type 2 diabetes subjects, and its relation to glycosylated haemoglobin (HbA1c), body mass index (BMI) and serum total cholesterol.

Aim and Objective- To study the incidence of left ventricular diastolic dysfunction (LVDD) and its correlation with HbA1c in young ( $<40$ years) normotensive, newly diagnosed type 2 diabetic patients.

\section{MATERIALS AND METHODS}

This cross-sectional study was done in rural Bangalore on 160 young ( $<40$ years) patients of newly diagnosed (within 1 month) type 2 DM visiting the Medicine outpatient, Rajarajeswari Medical College, Bangalore. Patients with established type 2 diabetes and already taking antidiabetic treatment, cardiac diseases like valvular heart disease, ischaemic and hypertensive heart disease, congestive heart failure, cardiomyopathy, renal failure, chronic pulmonary disease, severe anaemia and haemoglobinopathies were excluded from the study. These patients were informed about the study and informed consent was obtained before proceeding with the investigations. Patients selected were evaluated with relevant investigations like body mass index (BMI), fasting blood sugar, HbA1c level, total cholesterol and 2D echocardiography to assess LVDD. These selected patients were divided into 2 groups; one with left ventricular diastolic dysfunction (LVDD) and second group of subjects without LVDD. Various parameters like HbA1c, body mass index and serum total cholesterol were evaluated between these 2 groups. Statistical analysis was performed using SPSS software version 16.

\section{RESULTS}

Out of 160 patients, 114 were males and 46 were females. Baseline characteristics as in Table 1 . Mean age of the population was $35.79 \pm 2.8$ years, mean BMI were $26.15 \pm 2.7 \mathrm{~kg} / \mathrm{m} 2$, mean $\mathrm{HbA} 1 \mathrm{c}$ was $7.9 \pm 1.24$ and mean serum total cholesterol were $250.43 \pm$ 37.3. Overall incidence of LVDD was $31.2 \%$. Grade 1 LVDD was the most common. Mean level of HbA1c, BMI, serum total cholesterol and LVDD group was found statistically higher as compared to those without LVDD.

\section{CONCLUSION}

Nearly one-third of newly diagnosed type II diabetic patients were found to have diastolic dysfunction independent of confounding effect of hypertension and ischaemia. Most of the newly diagnosed diabetes with morbid obesity and high HbA1c level were found to have LV diastolic dysfunction irrespective of the age. HbA1c and BMI were found to be strong indicators of LVDD in young newly diagnosed cases of Type 2 DM, both in male and female sex. HbA1c emerges as an important indicator of diastolic dysfunction in early onset diabetes population in the study.

\section{KEYWORDS}

Diabetes, Body Mass Index, Cholesterol, Left Ventricular Diastolic Dysfunction.

HOW TO CITE THIS ARTICLE: Raj RAMR, Kothari YK, Kenchappa K, et al. Study of diastolic dysfunction in young (< 40 years) newly detected diabetes and its correlation with obesity, glycaemic levels and dyslipidaemia. J. Evolution Med. Dent. Sci. 2018;7(03):342-345, DOI: $10.14260 / \mathrm{jemds} / 2018 / 76$

'Financial or Other Competing Interest': None.

Submission 09-11-2017, Peer Review 26-12-2017,

Acceptance 04-01-2018, Published 13-01-2018.

Corresponding Author:

Dr. Ram Anil Raj M. R,

27/2, Heart and Rhythm Clinic,

$7^{\text {th }}$ Main, $2^{\text {nd }}$ Block,

Near Ashoka Pillar Canara Bank Branch,

Jayanagar, Bengaluru-560011.

E-mail: drramanilraj2001@yahoo.com

DOI: $10.14260 /$ jemds $/ 2018 / 76$

\section{BACKGROUND}

Diabetes mellitus (DM) is a common endocrine disorder affecting around 387 million people worldwide. ${ }^{1}$ The incidence of diabetes mellitus (DM) is increasing worldwide and rapidly assuming epidemic proportions. The Indian Council of Medical Research-Indian Diabetes Study (ICMRINDIAB), a national DM study estimates that currently India has 62.4 million people with DM. ${ }^{2}$ The majority (> 90\%) of them have Type 2 DM (T2DM). Over the last three decades, a number of epidemiological, clinical and autopsy studies have proposed the presence of diabetic heart disease as a distinct clinical entity. Diastolic heart failure (HF) is also referred to 
as HF with preserved left ventricular systolic function. The mortality rates among the patients with DHF ranges from 5\%-8\% annually as compared with $10 \%-15 \%$ among patients with systolic heart failure. ${ }^{3}$ Many studies have reported that the incidence of heart failure in diabetic subjects is high even in the absence of hypertension and coronary artery disease. Studies have reported a high prevalence of pre-clinical diastolic dysfunction among subjects with DM. 4 The evidence indicates that myocardial damage in diabetic subjects affects diastolic function before the systolic function. The pathogenesis of this left ventricular (LV) dysfunction in diabetic subjects is not clearly understood. Diabetic cardiomyopathy has been proposed as an independent cardiovascular disease, and many mechanisms such as microvascular disease, autonomic dysfunction, metabolic disorders and interstitial fibrosis have been suggested as causative factors. ${ }^{5}$ However, the exact aetiopathogenesis of diabetic cardiomyopathy still remains unclear. So far very few population-based studies have been carried out in India to demonstrate the prevalence of diastolic dysfunction in diabetic subjects in the Indian patients, that too in young newly detected diabetics. The objective of our study was to determine the incidence of asymptomatic LV diastolic dysfunction in young ( $<40$ years) newly diagnosed normotensive cases of type $2 \mathrm{DM}$ subjects and its relation to $\mathrm{HbA1c}$, age at the time of diagnosis, BMI and serum total cholesterol.

\section{Aims and Objectives}

To study the incidence of LVDD in young (age $<40$ years) normotensive, newly diagnosed type 2 DM patients by using 2D echocardiography and finding out its correlation with HbA1c, body mass index and serum total cholesterol level. This cross-sectional study was conducted in Rajarajeswari Medical College and Hospital, Bengaluru, India over a period of two years from May 2014 to June 2016. The study comprised a total of 160 cases of newly diagnosed (within 1 month) type $2 \mathrm{DM}$ under the age of 40 years including both males and females who clinically had no symptoms of cardiovascular involvement and blood pressure $<140 / 80$ mmHg with normal ECG. The diagnosis of diabetes was made on the basis of clinical evaluation, biochemical and ancillary investigation like fasting plasma glucose (FPG)/ postprandial plasma glucose (PPPG) and HbA1c according to recent American Diabetic Association (ADA) recommendations. A detailed clinical history with specific reference to cardiovascular symptoms, drug intake and smoking was taken. A complete general and systemic examination, particularly for stigmata of cardiovascular status was carried out. All patients with already diagnosed type $2 \mathrm{DM}$ and taking antidiabetic treatment, cardiac diseases like valvular heart disease, ischaemic and hypertensive heart disease, congestive heart failure, cardiomyopathy, renal failure, chronic pulmonary disease, severe anaemia and haemoglobinopathies were excluded from the study. Patients underwent thorough clinical examination supported by relevant investigations like blood glucose on admission, FPG/PPPG, renal function tests including electrolytes, fasting lipid profile (FLP), ECG, routine urine and microscopy study, fundoscopy and chest radiography. HbA1c was estimated by Boronate affinity chromatography, which separates total glycosylated haemoglobin by binding to solid-phase dihydroxyborate-13 using Nycocard immunoassay kit (USA).

\section{MATERIALS AND METHODS}

This cross-sectional study was done in rural Bangalore on 160 young ( $<40$ years) patients of newly diagnosed (within 1 month) type $2 \mathrm{DM}$ visiting the Medicine outpatient, Rajarajeswari Medical College, Bangalore. Patients with established type 2 diabetes and already taking antidiabetic treatment, cardiac diseases like valvular heart disease, ischaemic and hypertensive heart disease, congestive heart failure, cardiomyopathy, renal failure, chronic pulmonary disease, severe anaemia and haemoglobinopathies were excluded from the study. These patients were informed about the study and informed consent was obtained before proceeding with the investigations. Patients selected were evaluated with relevant investigations like body mass index (BMI), fasting blood sugar, HbA1c level, total cholesterol and 2D echocardiography to assess LVDD. These selected patients were divided into 2 groups; one with left ventricular diastolic dysfunction (LVDD) and second group of subjects without LVDD. Various parameters like HbA1c, body mass index and serum total cholesterol were evaluated between these 2 groups. Statistical analysis was performed using SPSS software version 16 .

All the subjects underwent resting transthoracic 2dimensional echocardiography and Doppler imaging to assess left ventricular diastolic function. Echocardiographer was not aware of this study to avoid bias in the interpretation. Echocardiography was performed by Philips echocardiography machine (5-1 MHz multi-frequency probe) according to the standard protocol. Pulsed-wave Doppler (PWD)- derived transmitral inflow velocities were obtained in the apical 4-chamber view with the sample volume placed at the mitral valve leaflet tips. Measurements included the transmitral early diastolic rapid filling (E-wave) and atrial contraction late filling (A-wave) velocities to calculate $\mathrm{E} / \mathrm{A}$ ratio, isovolumetric relaxation time (IVRT) and deceleration time (DT). For tissue Doppler imaging, the mitral annulus velocity was obtained with a $2 \mathrm{~mm}$ sample volume placed at the lateral side and septal side of the mitral annulus.

Diastolic dysfunction was labelled according to the standard guidelines. Left ventricular overall ejection fraction (systolic function) was calculated by modified Simpson's method and left ventricular ejection fraction (LVEF) $\geq 55 \%$ was considered as normal.6,7 All echocardiographic measurements were averaged over three consecutive cardiac cycles, measured by a single investigator blinded to all other variables. Left ventricular diastolic dysfunction was considered to be present if any of the following findings were seen as previously described-

1. E/A ratio $<1$ or $>2$,

2. DT $<150$ or $>220 \mathrm{~ms}$,

3. IVRT $<60$ or $>100 \mathrm{~ms}$, or

4. E/E ratio $>15$ ratio of mitral peak velocity of early filling (E) to early diastolic mitral annular velocity (E)\}.

\section{Classification of LVDD}

Grade 1: Delayed relaxation time, i.e. $\mathrm{E} / \mathrm{A}<1$.

Grade 2: Pseudonormalisation.

Grade 3: Reversible restrictive pattern.

Grade 4: Irreversible restrictive pattern. 


\section{Statistical Analysis}

Data was analysed for mean, percentage, standard deviation, chi-square test, multiple correlation and multivariate analysis by using SPSS-16 (Statistical Package for the Social Sciences) for Windows (SPSS, Chicago, IL). Variables that were not normally distributed were reciprocally transformed for analysis. The ' $\mathrm{t}$ ' test and Chi-square tests were applied to study quantitative and qualitative data respectively with ' $\mathrm{P}$ ' value $<0.05$ was considered statistically significant. Correlation of various factors was determined using $\mathrm{R}^{2}$ and multiple linear regression analysis. Correlation ( $\mathrm{r}$ ) findings were described as follows: $r=0.8$ (high correlation coefficient); $r=0.4-0.7$ (moderate correlation); and $r=0.3$ and above (low correlation coefficient).

\section{RESULTS}

Out of 160 patients, 114 were males and 46 were females. Baseline characteristics as in Table 1 . Mean age of the population was $35.79 \pm 2.8$ years, mean BMI were $26.15 \pm 2.7$ $\mathrm{kg} / \mathrm{m} 2$, mean $\mathrm{HbA} 1 \mathrm{c}$ was $7.9 \pm 1.24$ and mean serum total cholesterol were $250.43 \pm 37.3$ Overall incidence of LVDD was $31.2 \%$ (50/160). Grade 1 LVDD was the most common. Mean level of HbA1c, BMI and serum total cholesterol in LVDD group was found statistically higher as compared to those without LVDD as shown in Table 2.

\begin{tabular}{|c|c|c|}
\hline Characters & Range & Mean + SD \\
\hline Age & $29-40$ (in years) & $35.79 \pm 2.8($ in years $)$ \\
\hline Body Mass Index & $\begin{array}{c}21.6-33.6 \\
(\mathrm{~kg} / \mathrm{m} 2)\end{array}$ & $\begin{array}{l}26.15 \pm 2.7 \\
(\mathrm{in} \mathrm{kg} / \mathrm{m} 2)\end{array}$ \\
\hline $\begin{array}{l}\text { Serum Total } \\
\text { Cholesterol }\end{array}$ & $\begin{array}{c}180-332 \\
(\mathrm{mg} / \mathrm{dL})\end{array}$ & $\begin{array}{c}250.43 \pm 36.9 \\
\text { (in mg/dL) }\end{array}$ \\
\hline HbA1c & $\begin{array}{c}6.6-11.4 \\
\text { (in } \%)\end{array}$ & $\begin{array}{c}7.97 \pm 1.24 \\
\text { (in } \%)\end{array}$ \\
\hline
\end{tabular}

\begin{tabular}{|c|c|c|c|}
\hline Characteristics & $\begin{array}{c}\text { LVDD } \\
\text { Absent }\end{array}$ & $\begin{array}{c}\text { LVDD } \\
\text { Present }\end{array}$ & P Value \\
\hline Age & $36.17 \pm 2.3$ & $34.9 \pm 3.6$ & 0.12 \\
\hline BMI & $24.83 \pm 1.8$ & $29.02 \pm 2.0$ & $<0.01$ \\
\hline HbA1c & $7.3 \pm 0.5$ & $9.4 \pm 1.1$ & $<0.01$ \\
\hline $\begin{array}{c}\text { Serum Total } \\
\text { Cholesterol }\end{array}$ & $239.05 \pm 31.7$ & $275.4 \pm 35.6$ & $<0.01$ \\
\hline
\end{tabular}

Table 2. Comparison of Means of Age, BMI, HbA1c and Serum Total Cholesterol according to Presence of LVDD

\section{Correlation of LVDD with Respect to Obesity, Glycaemic} Level and Dyslipidaemia

Out of 50 patients who had diastolic dysfunction, none were in normal BMI range, i.e. $<23.5 \mathrm{~kg} / \mathrm{m} 2$. As mentioned in Table 3 , all morbid obese subjects had LV diastolic dysfunction. Near half $(51.5 \%)$ of obese subjects had LV diastolic dysfunction. Obesity was a positive correlate with LV diastolic dysfunction with $\mathrm{R}$ squared value $=0.533$ (with ' $\mathrm{p}$ ' $<0.01$ ).

\begin{tabular}{|c|c|c|}
\hline $\begin{array}{c}\text { BMI Range } \\
\text { (in kg/m2) }\end{array}$ & $\begin{array}{c}\text { LVDD Absent } \\
\text { (N/\%) }\end{array}$ & $\begin{array}{c}\text { LVDD Present } \\
\text { (N/\%) }\end{array}$ \\
\hline Normal $(<23.5)$ & $23 / 23(100 \%)$ & $0 / 23(0 \%)$ \\
\hline Overweight $(23.5-25.0)$ & $55 / 57(96.5 \%)$ & $2 / 57(3.5 \%)$ \\
\hline Obese $(25.0-30.0)$ & $32 / 66(48.5 \%)$ & $34 / 66(51.5 \%)$ \\
\hline Morbid Obese $(>30.0)$ & $0 / 14(0 \%)$ & $14 / 14(100 \%)$ \\
\hline \multicolumn{2}{|c|}{ Table 3. Correlation of LVDD with BMI } \\
\hline
\end{tabular}

As shown in Table 4, all subjects who had very high HbA1c levels (> 9.0\%) had LV diastolic dysfunction. Glycaemic level based on HbA1c levels were positively correlated with $\mathrm{LV}$ diastolic dysfunction with $\mathrm{R}$ squared value $=0.687(\mathrm{p}<0.01)$

\begin{tabular}{|c|c|c|}
\hline $\begin{array}{c}\text { HbA1c Range } \\
\text { (in \%) }\end{array}$ & $\begin{array}{c}\text { LVDD Absent } \\
\text { (N (\%) }\end{array}$ & $\begin{array}{c}\text { LVDD Present } \\
\text { (N (\%) }\end{array}$ \\
\hline $6.5-7$ (good) & $46 / 46(100 \%)$ & $0 / 46(0 \%)$ \\
\hline $7.0-8.0$ (average) & $46 / 50(92 \%)$ & $4 / 50(8 \%)$ \\
\hline $8.0-9.0$ (poor) & $18 / 31(58 \%)$ & $13 / 31(42 \%)$ \\
\hline$>9.0$ (very poor) & $0 / 33(0 \%)$ & $33 / 33(100 \%)$ \\
\hline \multicolumn{2}{|c|}{ Table 4. Correlation of LVDD with HbA1c } \\
\hline
\end{tabular}

\section{DISCUSSION}

Our study demonstrates that nearly one-third of newly detected diabetes, young and normotensive individuals have asymptomatic diastolic dysfunction. Patil et al in their study of 127 asymptomatic subjects found the prevalence of diastolic dysfunction in asymptomatic type 2 diabetics as high as $54.33 \% .{ }^{8}$ Higher prevalence could be attributed to the duration of diabetes in these patients, because unlike our study cases of diabetes were already diagnosed and were of more than 5 years duration. Absence of cases with systolic dysfunction signifies that diastolic dysfunction is the earliest marker of diabetic cardiomyopathy, which precedes systolic dysfunction. Predictors for LV diastolic dysfunction in these individuals are $\mathrm{HbA1c}$, body mass index and higher serum total cholesterols. Diabetes is presumed to increase stiffness through myocardial deposition of collagen and advanced glycation end products. Masugata et $\mathrm{al}^{9}$ in their case control study of 77 normotensive patients found that the cardiac diastolic dysfunction without LV systolic dysfunction in patients with well-controlled type $2 \mathrm{DM}$ is related neither to hypertension nor LV hypertrophy, but rather to aging and the duration of type 2 DM. CM Schannwell et al 10 in their study population of 87 subjects concluded that even young subjects with diabetes mellitus suffer from a diastolic dysfunction, while systolic ventricular function is normal. Hameedullah et $\mathrm{al}^{11}$ in their study population of 60 patients with type $2 \mathrm{DM}$ found that there was strong correlation between $\mathrm{HbA1c}$ level and diastolic indices (' $\mathrm{P}$ ' < 0.05). Diastolic dysfunction was more frequent in poorly controlled diabetic patients and its severity is correlated with glycaemic control. Similarly, in our study, all subjects with $\mathrm{HbA1c}>9 \%$ had diastolic dysfunction compared to none if $\mathrm{HbA} 1 \mathrm{c}<7.0 \%$. Celentano et al ${ }^{12}$ also studied subjects with normal glucose tolerance with impaired glucose tolerance and with type $2 \mathrm{DM}$ and found early signs of diastolic dysfunction (assessed by E/A mitral flow ratio), not only in patients with diabetes but also in those with impaired glucose tolerance, independent of the confounding role of ischaemia, body weight and blood pressure. Holzmann et $\mathrm{al}^{13}$ showed in a middle-aged population without previously diagnosed DM, a continuous relationship between concentrations of fasting plasma glucose, HbA1c and LVDD. So, the present study confirmed previous findings. Therefore, future studies should be conducted to test the hypothesis that screening and aggressive management of diabetic patients with pre-clinical diastolic dysfunction may delay the progression to heart failure. 


\section{Study Limitation}

One of the limitations of this study was the population studied were South Indian population who are mostly on rice-based food, which has high glycaemic index. Study population was only 160 . Thus, these findings need to be examined in different racial and ethnic groups and in large number.

\section{CONCLUSION}

Nearly one-third of newly diagnosed type 2 diabetic patients were found to have diastolic dysfunction independent of confounding effect of hypertension and ischaemia. Most of the newly diagnosed diabetes with morbid obesity and high HbA1c levels were found to have LV diastolic dysfunction irrespective of the age.

HbA1c and BMI were found to be strong indicators of LVDD in young newly diagnosed cases of Type $2 \mathrm{DM}$, both in male and female sex.

HbA1c emerges as an important indicator of diastolic dysfunction in early onset diabetes population in the study.

\section{REFERENCES}

[1] Shi Y, Hu FB. The global implications of diabetes and cancer. Lancet 2014;383(9933):1947-8.

[2] Anjana RM, Pradeepa R, Deepa M, et al. Prevalence of diabetes and prediabetes (impaired fasting glucose and/or impaired glucose tolerance) in urban and rural India: phase I results of the Indian Council of Medical Research-INdia DIABetes (ICMR-INDIAB) study. Diabetologia 2011;54(12):3022-7.

[3] Piccini JP, Klein L, Gheorghiade M, et al. New insights into diastolic heart failure: role of diabetes mellitus. Am J Med 2004;116(Suppl 5A):64S-75S.

[4] Kazik A, Wilczek K, Poloński L. Management of diastolic heart failure. Cardiol J 2010;17(6):558-65.
[5] From AM, Scott CG, Chen HH. Changes in diastolic dysfunction in diabetes mellitus over time. Am J Cardiol 2009;103(10):1463-6.

[6] Ommen SR, Nishimura RA, Appleton CP, et al. Clinical utility of Doppler echocardiography and tissue Doppler imaging in the estimation of left ventricular filling pressures: a comparative simultaneous Doppler-catheterization study. Circulation 2000;102(15):1788-94.

[7] Oh JK, Seward JB, Tajik AJ. Assessment of diastolic dysfunction and diastolic heart failure. In: Oh JK, (eds). The echo manual. $3^{\text {rd }}$ edn. New Dehli: Wolters Kluwer, 2006:120-41.

[8] Patil VC, Patil HV, Shah KB, et al. Diastolic dysfunction in asymptomatic type 2 diabetes mellitus with normal systolic function. J Cardiovasc Dis Res 2011;2(4):21322.

[9] Masugata H, Senda S, Goda F, et al. Left ventricular diastolic dysfunction in normotensive diabetic patients in various age strata. Diabetes Res Clin Pract 2008;79(1):91-6.

[10] Schannwell CM, Schneppenheim M, Perings S, et al. Left ventricular diastolic dysfunction as an early manifestation of diabetic cardiomyopathy. Cardiology 2002;98(1-2):33-9.

[11] Hameedullah, Faheem M, Bahadar S, et al. Effect of glycaemic status on left ventricular diastolic function in normotensive type 2 diabetic patients. J Ayub Med Coll Abbottabad 2009;21(3):139-44.

[12] Celentano A, Vaccaro O, Tammaro P, et al. Early abnormalities of cardiac function in non-insulindependent diabetes mellitus and impaired glucose tolerance. Am J Cardiol 1995;76(16):1173-6.

[13] Holzmann M, Olsson A, Johansson J, et al. LV diastolic function is related to glucose in a middle-aged population. J Intern Med 2002;251(5):415-20. 